\title{
Adult Intussusception Caused by an Intestinal Lipoma: Report of a Case
}

\author{
Ichiro Akagi ${ }^{1}$, Masao Miyashita ${ }^{1}$, Masayoshi Hashimoto², \\ Hiroshi Makino ${ }^{1}$, Tsutomu Nomura ${ }^{1}$ and Takashi Tajiri ${ }^{1}$ \\ ${ }^{1}$ Surgery for Organ Function and Biological Regulation, Graduate School of Medicine, Nippon Medical School \\ ${ }^{2}$ Department of Surgery, Mitsubishi Ohkurayama Hospital, Kanagawa
}

\begin{abstract}
Intussusception in adults represents only $5 \%$ of all cases and is usually caused by a small bowel lesion acting as the apex of the intussusception. We report an unusual case of intussusception in man caused by a lipomatous lesion located in the ileum acting as the lead point. After repeated admissions to our hospital for ileus, the possibility of intussusception was finally raised by a computed tomographic scan of the abdomen. The patient underwent primary resection of the intussuscepted intestine, which resulted in a long-lasting resolution of the symptoms. The resected specimen contained a round tumor measuring $27 \times 27 \times 40 \mathrm{~mm}$ which was diagnosed histopathologically as an intestinal lipoma. This case highlights the uncommon causation of adult intussusception by an intestinal lipoma.
\end{abstract}

(J Nippon Med Sch 2008; 75: 166-170)

Key words: intussusception, ileal lipoma, adult, intestine

\section{Introduction}

Intussusception occurs when a proximal segment of the bowel (intussusceptum) telescopes or invaginates into the lumen of the adjacent distal segment (intussuscipiens). Although the condition is common in children, intussusception in adults accounts for only $1 \%$ of intestinal obstructions in ${ }^{1}$ and $5 \%$ of all intussusceptions ${ }^{2}$. In adult intussusception, the presence of malignant small bowel tumors acting as the apex of intussusception must always be suspected, although benign smallbowel lesions could be the underlying cause ${ }^{3,4}$. Moreover, the diagnosis is often difficult because of the lack of specific clinical signs and of the problems in examining the small intestine. Lipoma, which frequently occurs in the terminal ileum, is the second most common benign tumor of the small intestine and tends to cause intussusception. Here, we report a surgical case of intussusception due to an intestinal lipoma.

\section{Case Report}

A 36-year-old man with no previous illnesses or relevant family history was admitted to our hospital. He complained of colicky epigastric pain, which was of variable severity, aggravated by food ingestion, and associated with nausea, vomiting, and abdominal distension. A plain X-ray film of the abdomen revealed the formation of a niveau, confirming bowel obstruction. The patient was found to have ileus, and treated with enemas and suppositories. After

Correspondence to Ichiro Akagi, MD, Department of Surgery (Divisions of Gastroenterology, General, Breast and Transplant), Nippon Medical School, 1-1-5 Sendagi, Bunkyo-ku, Tokyo 113-8603, Japan

E-mail: ichiro@nms.ac.jp

Journal Website (http://www.nms.ac.jp/jnms/) 


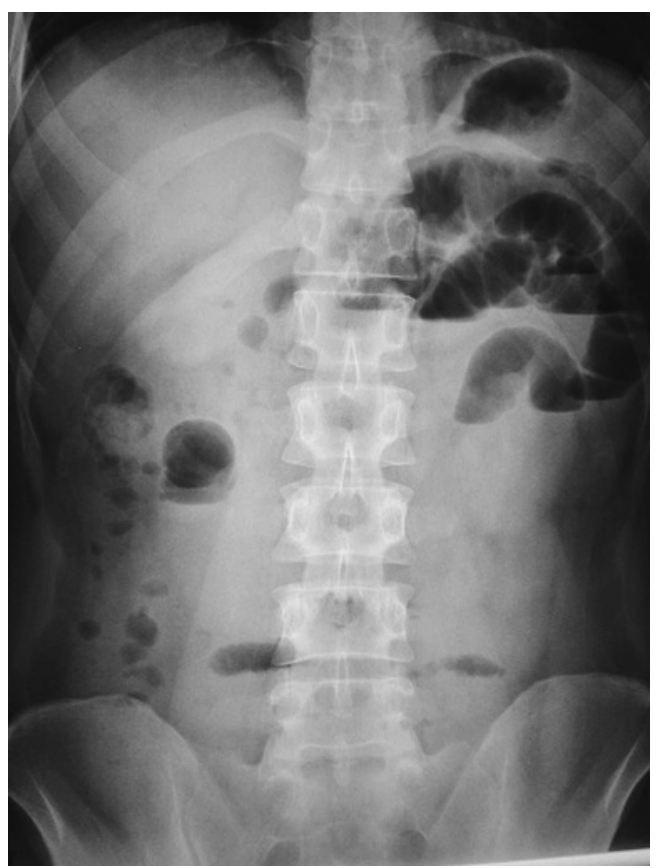

Fig. 1 Abdominal radiography revealed a prominently dilated small bowel with some air-fluid levels.

two weeks of hospitalization, his symptoms were relieved and he was discharged. However, he was readmitted one week later with similar symptoms.

At the second admission, the patient showed pallor and a distended abdomen with no palpable mass. He only had mild tenderness in the lower abdomen. Bowel sounds were normal, and rectal examination revealed an empty rectum with no sign of bleeding. Laboratory studies showed a white blood cell count of $10,900 / \mu \mathrm{L}$ and a serum Creactive protein level of $3.1 \mathrm{mg} / \mathrm{dL}$. Results of all other laboratory studies, including measurement of serum electrolytes levels and urinalysis, were within normal limits. Abdominal radiography revealed prominent dilatation of the small bowel with air-fluid levels (Fig. 1). Although abdominal ultrasonography (US) did not provide any useful clues to the diagnosis, computed tomography (CT) of the abdomen revealed findings indicative of ileal intussusception, as a target-like (bowel-in-bowel) mass with fat density, depending on the angle of the CT beam relative to the axis of the intussusception (Fig. 2). Therefore, the patient was treated by means of a long tube. A gastrograffin contrast X-ray study performed via the long tube revealed dilatation of

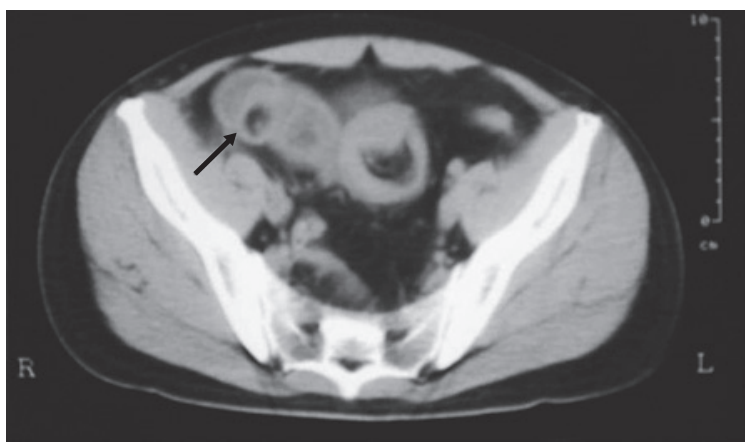

Fig. 2 CT scan showed findings indicative of an ileal intussusception as a target-like mass. A round mass of fat density representing lipoma $(\rightarrow)$ was detected within the lumen of the intussuscipiens.

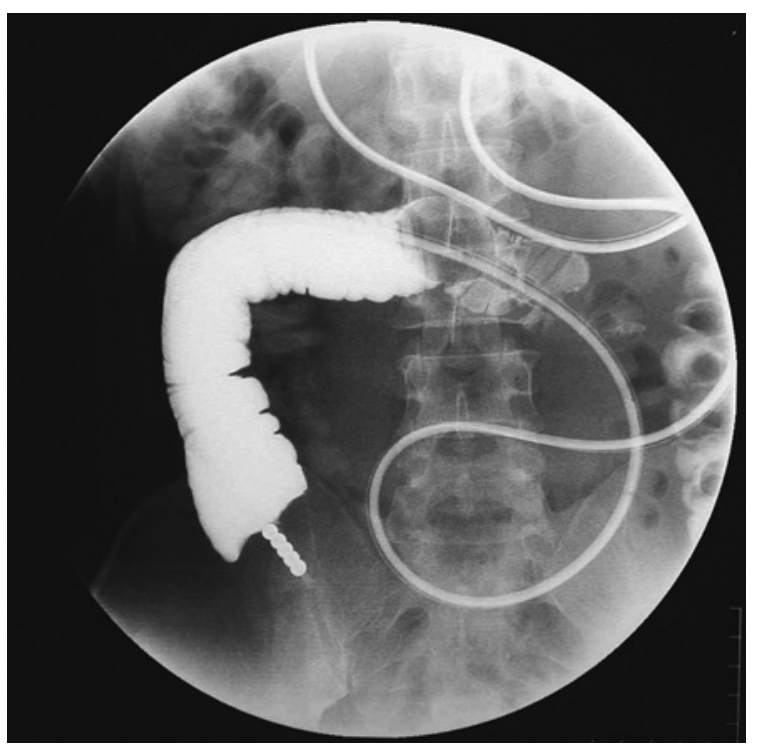

Fig. 3 A gastrograffin contrast X-ray film revealed a dilated proximal intestine, a filling defect $27 \mathrm{~mm}$ long and $27 \mathrm{~mm}$ wide, and a narrow central canal sign in the jejunum.

the proximal small intestine, a filling defect that was $27 \mathrm{~mm}$ long and $27 \mathrm{~mm}$ wide and a narrrow central canal sign in the ileum (Fig. 3). These findings were strongly suggestive of an intestinal tumor with intussusception. The symptoms were somewhat relieved by withholding oral intake and administration of intravenous fluids, and elective surgery was scheduled after admission. Laparotomy revealed an ileal intussusception caused by a mass in the ilem. There was no apparent lymphadenopathy. We performed primary resection of the intussuscepted intestine $80 \mathrm{~cm}$ proximal to Bauhin's valve. Approximately $12 \mathrm{~cm}$ of small 


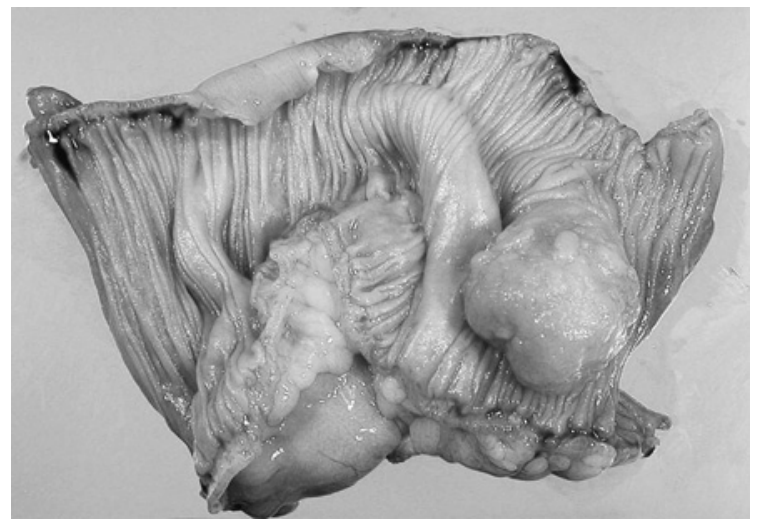

Fig. 4 Operative specimen showing a lipomatous lesion measuring $27 \times 27 \times 40 \mathrm{~mm}$, which acted as a lead point for the ileocolic intussusception.

intestine, including the tumor, was resected after which an end-to-end anastomosis was performed. The patient had an uneventful recovery. He passed flatus on postoperative day 1 , began to receive oral fluids on day 2, and was discharged on day 15. At the last follow-up examination performed 2 years after the surgery, the patient was free of bowel symptoms.

Gross examination of the resected specimen revealed a reddish mass measuring $27 \times 27 \times 40 \mathrm{~mm}$ without ulceration or any evidence of malignancy (Fig. 4). Histological examination revealed a large polypoid lipoma of the ileum, which was thought to have arisen from the submucosal layer (Fig. 5).

\section{Discussion}

Tumors of the small bowel account for only $1 \%$ to $2 \%$ of all gastrointestinal tumors, and benign tumors account for approximately 30\% of all small-bowel tumors. Gastrointestinal stromal tumors are the most common symptomatic benign tumors of the small bowel, and lipomas are the second most common. According to our review of the literature, the symptoms of lipoma include abdominal pain (68\%), intussusception (44\%), hemorrhage (29\%), vomiting $(24 \%)$, and obstructive symptoms $(18 \%)^{5}$. Of the cases reviewed, $83 \%$ were in the ileal region, and $75 \%$ of these are found within $60 \mathrm{~cm}$ of Bauhin's valve $^{5}$. In the present case, the lipoma was located $80 \mathrm{~cm}$ proximal to Bauhin's valve.

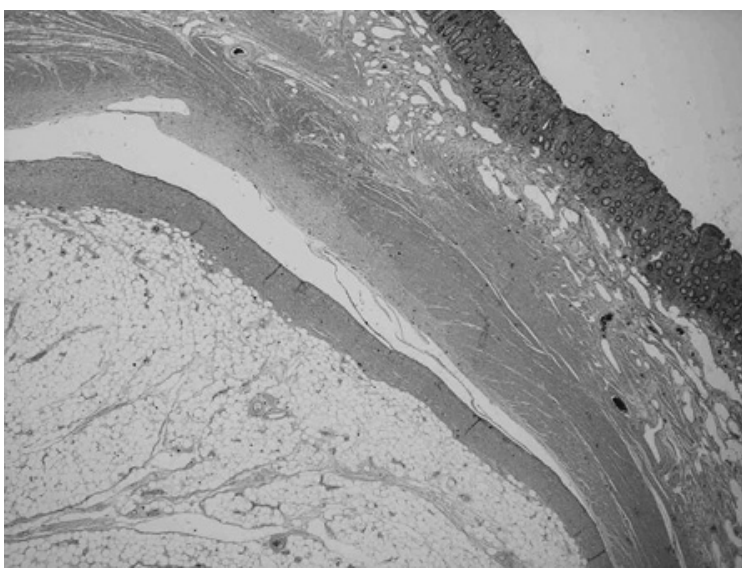

Fig. 5 Histopathologic examination of the tumor revealed fat cells proliferating in the submucosal layer (hematoxylin and eosin, $\times 20$ ).

Intussusception in adults is usually caused by a tumor that acts as the lead point and is commonly located at or near the ileocecal valve. Whereas the diagnosis is usually already suspected in children before imaging, in adults, the diagnosis is often made unexprectedly and the presence of a malignancy as the leading point must first be excluded. However, it is estimated that in $52 \%$ of cases in adults, intussusception is caused by benign small bowel conditions ${ }^{4}$, such as Peutz-Jeghers polyp ${ }^{6}$, Meckel's diverticulum $^{7}$, and tubulovillous adenoma of the ileocecal valve ${ }^{8}$. In contrast, intussusception is seen in only $5 \%$ of patients with leiomyoma ${ }^{9}$. Lipoma is thought to arise from the mucinous layer, whereas leiomyoma arises from the muscle layer, which perhaps explains why lipomas tend to invaginate into the intestine. In the present case, histological examination revealed a polypoid lipoma of the ileum, which was thought to have arisen from the submucosal layer. In general, intussusception associated with lipomas develops rapidly and patients tend to recover spontaneously.

Because of the nonspecific clinical presentation, tumors of the small intestine can be difficult to diagnose and a correct preoperative diagnosis is made in only about $32 \%$ of cases. Hence, the diagnosis of intussusception in these cases can often not be established preoperatively, and the condition is often only confirmed at laparotomy. CT, which sometimes obviates the need for further radiological 
studies, and US are of great help in the diagnosis of intussusception. In our case, CT showed the lipoma as a homogeneous intraluminal mass with attenuation values between -80 and $-120 \mathrm{HU}$, consistent with fat, and intussusception was seen as a concentric pattern in the intestinal walls ${ }^{10,11}$. Other investigators have reported that it is possible to distinguish lipomas from liposarcomas on CT scans, which show the interior of a liposarcoma as being nonhomogeneous, with a higher density than lipomas ${ }^{12}$. US should be performed because it is a fast, noninvasive, easy to perform, and reproducible. The most common US feature of intussusception is the "target" sign. Sometimes, its diagnostic value is undermined in the presence of acute obstructive symptoms because of the poor signal transmission caused by the presence of too much bowel air ${ }^{13}$. This may explain the difficulty in diagnosing the intussusception by abdominal US in our patient. In children, barium enema is often used to diagnose and treat intussusception; however, it is rarely performed in adults. This is because false positives are more common in adults with masses, which sometimes simulate an intussusception. Therefore, barium enema was not performed during any of our patient's previous hospital admissions.

Surgical intervention is indicated in all cases of adult intussusception, because most of these cases are caused by mass lesions. Controversy remains about whether reduction of the intussusception at operation is necessary. Some investigators recommend "en bloc" resection of this friable, edematous bowel loop. Because the likelihood of an intestinal tumor is high in adults with intussusceptions and because $24 \%$ of small intestinal tumors are malignant, some believe that reduction should not be attempted ${ }^{13}$. Alternatively, it is now accepted that gentle operative reduction can be attempted safely before resection, to avoid unnecessary excision of healthy bowel ${ }^{8}$. The length of the intestine involved in the intussusception varies from a few centimeters to several tens of centimeters. Although attempts at operative reduction used to be discouraged for fear of tumor dissemination, this risk is only a theoretical, and some investigators believe that gentle reduction should be attempted in selected cases ${ }^{14}$. Without reduction, operative resection of a long segment of intussusception often necessitates excision of a longer segment of the intestine than necessary, which may compromise the mesenteric vessels. We did not attempt reduction in our patient because of the short length of the intestine involved in the intussusception.

Recently, the use of laparoscopic surgery for benign bowel tumors and ileocecal intussusception has increased ${ }^{15-18}$. However, intestinal dilatation was present in our case, and performing laparoscopic surgery in the presence of this finding was considered difficult. Therefore, we believe that small laparotomy, rather than laparoscopic surgery, was the optimal approach in our case.

In conclusion, this is a report of a case of intussusception in an adult with an unusually benign cause, which was finally diagnosed with $\mathrm{CT}$ and treated with surgical resection.

\section{References}

1. Stubenbord WT, Thorbjarnarson B: Intussusception in adults. Ann Surg 1970; 1722: 306-310.

2. Nagorney DM, Sarr MG, McIlrath DC: Surgical management of intussusception in the adult. Ann Surg 1981; 1932: 230-236.

3. Desai N, Wayne MG, Taub PJ, Levitt MA, Spiegel R, Kim U: Intussusception in adults. Mt Sinai J Med 1999; 66: 336-340.

4. Azar T, Berger DL: Adult intussusception. Ann Surg 1997; 2262: 134-138.

5. Yao T YK, Matake H, Furukawa K, Nagae T, Motomura A: Primary small intestinal tumors. Stomach Intestine 2001; 36: 871-881.

6. Meshikhes AW, Al-Saif O, Al-Otaibi M: Duodenal and ampullary obstruction by a Peutz-Jeghers polyp. Eur J Gastroenterol Hepatol 2000; 1211: 1239-1241.

7. Blakeborough A, McWilliams RG, Raja U, Robinson PJ, Reynolds JV, Chapman AH: Pseudolipoma of inverted Meckel's diverticulum:clinical, radiological and pathological correlation. Eur Radiol 1997; 76: 900-904.

8. Baig MK, Wise M, Wexner SD: Controversies in the treatment of adult long ileo-caecal intussusception. Am Surg 2000; 66: 742-743.

9. Muraoka M, Satoh H, Nakajima K, Yamazaki M, Ishikawa T: A case of intussusception by small intestinal lipoma. Jpn J Gastroenterol Surg 1989; 22: 2118-2122.

10. Urbano J, Serantes A, Hernandez L, Turegano F: Lipoma-induced jejunojejunal intussusception: US and CT diagnosis. Abdom Imaging 1996; 216: 522524 . 
11. Burckley JA: CT evalustion of small bowel neoplasm. Radiographics 1998; 18: 379-382.

12. Heiken JP, Gold RP: Computed tomography as a definitive method for diagnosing gastrointestinal lipomas. Radiology 1982; 142: 409-414.

13. Begos DG, Sandor A, Modlin IM: The diagnosis and management of adult intussusception. Am J Surg 1997; 1732: 88-94.

14. Reijnen HA, Joosten HJ, de Boer HH: Diagnosis and treatment of adult intussusception. Am J Surg 1989; 1581: 25-28.

15. Fumimoto Y, Hasegawa J, Nakajima K, Ito T, Matsuda H: A case of adult intussusception due to ileal lipoma diagnosed preoperatively and treated by laparoscopic assisted surgery. J Jpn Soc Endosc Surg 2005; 10: 416-419.

16. Chiu CC, Wei PL, Huang MT, Wang W, Chen TC,
Lee WJ: Laparoscopic treatment of ileocecal intussusception caused by primary ileal lymphoma. Surg Laparosc Endosc Percutan Tech 2004; 142: 9395.

17. Alonso V, Targarona EM, Bendahan GE, et al.: Laparoscopic treatment for intussusception of the small intestine in the adult. Surg Laparosc Endosc Percutan Tech 2003; 136: 394-396.

18. Tsushimi $\mathrm{T}$, Matsui $\mathrm{N}$, Kurazumi $\mathrm{H}$, et al: Laparoscopic resection of an ileal lipoma: Report of a case. Surg Today 2006; 3611: 1007-1011.

(Received, January 28, 2008)

(Accepted, February 29, 2008) 